\title{
Broadband SESAM for mode locked Yb:fiber lasers
}

\author{
CHEN LingLing ${ }^{1}$, ZHANG Meng $^{1}$, WANG Xi ${ }^{1}$, LI WenChao ${ }^{1}$, WEI Yun ${ }^{1}$, MA YunFeng ${ }^{2}$, \\ FAN ZhongWei ${ }^{2}$, NIU Gang ${ }^{2}$, YU Jin ${ }^{2}$, LIU Yang ${ }^{2}$, ZHANG Xue ${ }^{2} \&$ ZHANG ZhiGang ${ }^{1^{*}}$ \\ ${ }^{1}$ Institute of Quantum Electronics, State Key Laboratory of Advanced Optical Communication System and Networks, School of Electronics Engi- \\ neering and Computer Science, Peking University, Beijing 100871, China; \\ ${ }^{2}$ Academy of Opto-Electronics of Chinese Academy of Sciences, Beijing 100080, China
}

Received December 4, 2010; accepted February 15, 2011

\begin{abstract}
We report a semiconductor saturable absorber mirror (SESAM) with phase shift compensation. The SESAM shows a uniform low-intensity reflectivity and a modulation depth up to $12 \%$ for the wavelength from 1000 to $1100 \mathrm{~nm}$. A linear cavity all-normal dispersion $\mathrm{Yb}$-doped fiber laser has proved its mode locking performance for the wavelength of both $1030 \mathrm{~nm}$ and $1064 \mathrm{~nm}$.
\end{abstract}

Yb:doped fiber laser, semiconductor saturable absorber, mode locking

Citation: Chen L L, Zhang M, Wang X, et al. Broadband SESAM for mode locked Yb:fiber lasers. Chinese Sci Bull, 2011, 56: 1348-1351, doi: 10.1007/ s11434-011-4432-0

Semiconductor saturable absorbers (SESAMs) play the key role in highly stable, passively mode-locked ultrashort pulsed laser sources. Since 1992, extensive theoretical and experiment studies have been conducted on SESAM mode locked lasers [1-3].

As the demand for highly reliable passively mode-locked fiber lasers is increasing, highly reliable SESAMs become important. Unlike in solid-state lasers, mode locking in fiber lasers requires a high modulation depth. A simple solution to the high modulation depth is to increase the absorption layer thickness. However, there are two issues in the SESAMs in doing such way: the large variation in the reflectivity and the possible optical damage on the SESAMs. A thick absorption layer introduces a large phase shift that results in a modulation over the reflection band of the Bragg mirror [4,5], due to the imaginary part of the refractive index of the absorption material. The high absorption may also cause a high saturation fluence, which is the origin of the optical damage.

To avoid the optical damage on the SESAM, Grange et al. [6] realized that a low the saturation fluence is the key solution for the damage since the low saturation fluence

*Corresponding author (email: zhgzhang@pku.edu.cn) ensures the laser mode locking at relatively lower fluence, so that it never exceeds the damage threshold. They demonstrated that a thin layer can also show a relatively high modulation depth but with a saturation fluence as low as 1.1 $\mu \mathrm{J} / \mathrm{cm}^{2}$ [7]. The key point is to locate the absorption layer in the maximum electric field, as well as to apply an anti-reflection coating to enhance the field.

However, there has not been a solution reported for the thick absorption layer. The commercially available SESAMs with high modulation depth basically have a non-uniform low-intensity reflectivity with respect to wavelength, and a high saturation fluence $\left(>50 \mu \mathrm{J} / \mathrm{cm}^{2}\right)$. In this paper, we propose a phase shift compensated high modulation depth design of SESAM and demonstrate the uniform low intensity reflectivity and low saturation fluence. This SESAM has been verified in mode locking of a linear cavity all-normal dispersion Yb-doped fiber laser both in $1030 \mathrm{~nm}$ and $1064 \mathrm{~nm}$, respectively $[8,9]$.

\section{Design of the SESAM}

High modulation depth of a SESAM implies thick 
absorption layers. The conventional design of this kind of SESAM is simply to increase the absorption layer-InGaAs thickness. However, either our calculation or the data of commercial products show non-uniform curves.

The non-uniform curve can be brought by resonance of the layer structure, or in more cases the large absorption. This is recognized due to the complex index of the absorption layer which is in the form of $n=n_{0}-i \kappa$, where $n_{0}$ and $\kappa$ are the real and imaginary parts respectively. For the InAs, the imaginary part of the refractive index is estimated as 0.4 [10], while the real part is obtained by the linear fitting between InAs and GaAs. The function of the imaginary part is equivalent to a thinner layer than designed that shifts the resonant valley towards long wavelength direction, so that a resonant-like dip appears on the low reflection curve. In order to obtain the uniform and symmetric absorption, we must compensate for this phase shift. This can be made by increasing the thickness of absorption layer.

The structure of the SESAM with phase shift compensation is shown in Figure 1. The bottom Bragg mirror is 22-pair quarter wave GaAs/AlAs layers for the central wavelength $1035 \mathrm{~nm}$. Instead of one think layer of absorption, the absorption layer $\operatorname{In}_{0.3} \mathrm{Ga}_{0.7}$ As was divided into two parts; each has a thickness of $0.26 \lambda$. For locating the absorption layer in the peak of the electric field, the absorption layers are separated by three $\operatorname{In}_{0.3} \mathrm{Al}_{0.7} \mathrm{As}$ layers in the order from the top of $0.125 \lambda, 0.25 \lambda$ and $0.125 \lambda$. The total thickness of absorption layer is $0.52 \lambda$. The $\operatorname{In}_{0.3} \mathrm{Al}_{0.7} \mathrm{As}$ also serves as a strain-relaxation layer.

By assuming a constant imaginary part of the refraction index corresponding uniform absorption coefficient over wavelength, the calculated reflectivity is shown Figure 2. The calculated group delay dispersion (GDD) of the SESAM is also flat over $1010-1070 \mathrm{~nm}$ and is shown in Figure 3.

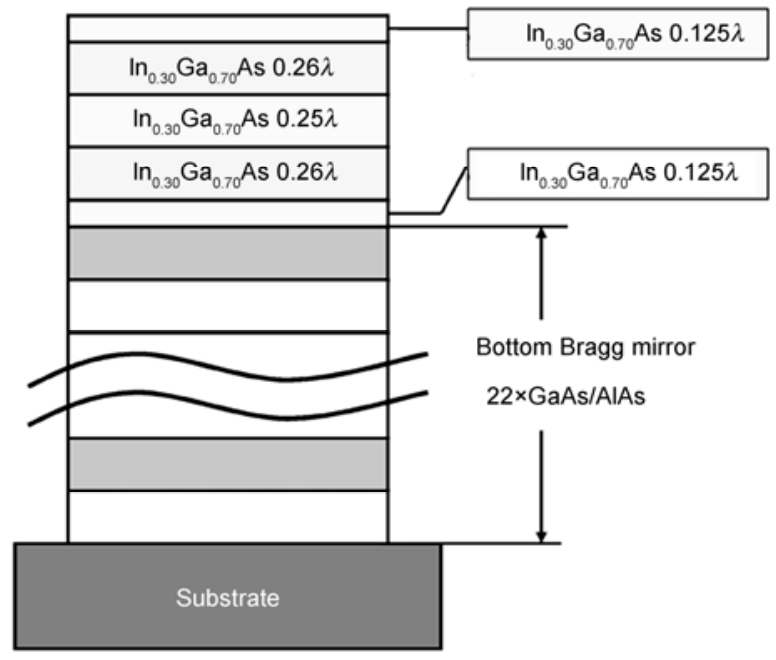

Figure 1 Structure of the multi-layer design with phase shift compensation.

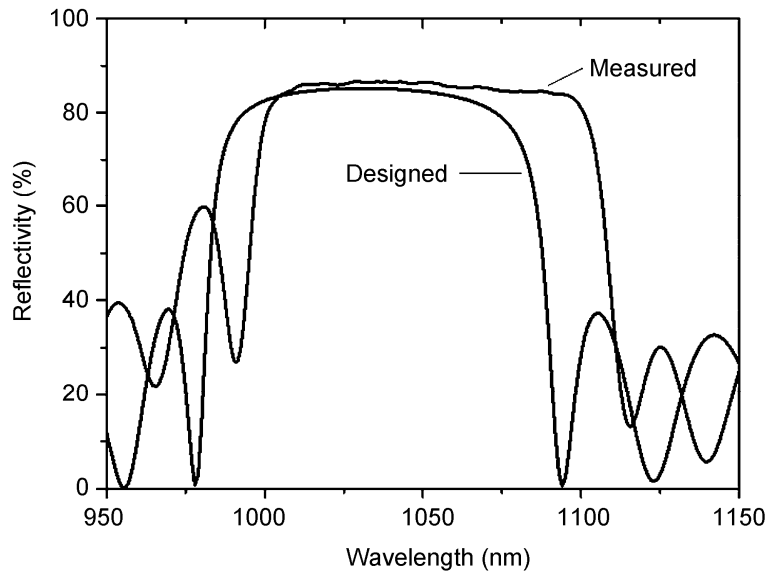

Figure 2 Designed and measured reflectivity of the SESAM design with phase shift compensation.

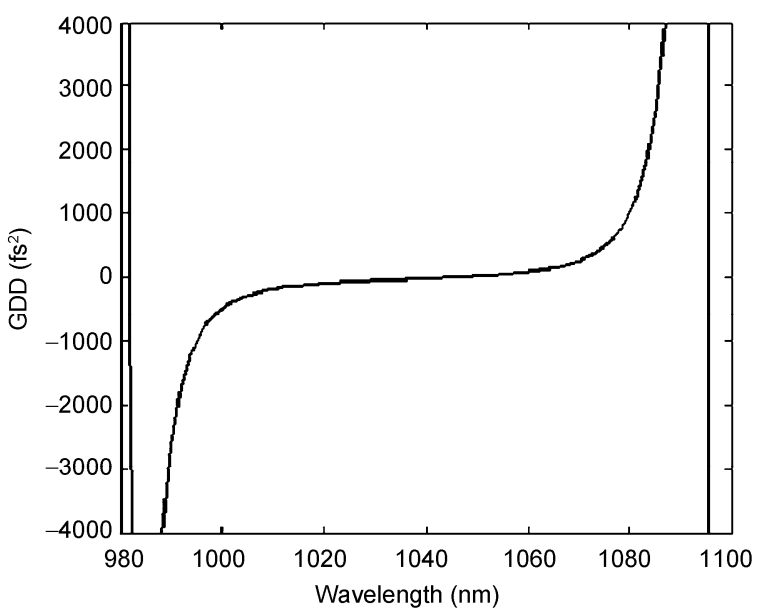

Figure 3 Calculated GDD of the SESAM with phase shift compensation.

The above SESAM was grown by molecular beam epitaxy at low temperature $\left(280^{\circ} \mathrm{C}\right)$ for fast recovery of the saturation. The recovery time is estimated to be less than 2 ps. The measured low intensity reflectivity is shown in Figure 3 and is compared with the designed. It is seen that the reflection is symmetric and uniform in wavelength 1000$1100 \mathrm{~nm}$.

\section{Measurement of the saturation fluence}

We determined the saturation fluence by measuring the nonlinear reflectivity. The measurement system consists of a pulsed laser source, a variable attenuator and the detection unit (Figure 4). The pulsed laser source is a mode locked $\mathrm{Yb}$ fiber laser with a pulse 140-fs long and a pulse energy up to $10 \mathrm{~J}$. The laser beam was divided into measurement and reference arms by a polarizing beam splitter cube (PBS). A lens with a focal length of $75 \mathrm{~mm}$ was applied before the SESAM to obtain a $4 \mu \mathrm{m}$ beam waist (radius) on the sample, 


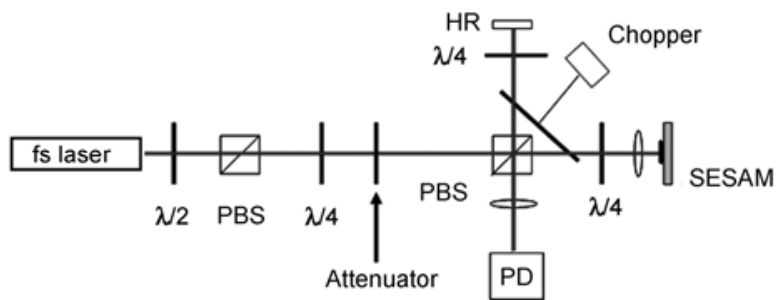

Figure 4 Measurement setup of the SESAM with phase shift compensation.

which allowed fluence up to hundreds of $\mu \mathrm{J} / \mathrm{cm}^{2}$. The signal separation in time was achieved by a chopper wheel which simultaneously chops both arms [11].

The measured reflectivity is shown in Figure 5. By fitting the data, we obtained the saturation fluence $F_{\text {sat }}<30.7 \mu \mathrm{J} / \mathrm{cm}^{2}$, the modulation depth $12 \%$, and the non-saturable loss $\Delta R_{\mathrm{ns}}$ $=3 \%$.

\section{Laser experiment}

The performance of the SESAM was experimentally verified with a ring cavity $\mathrm{Yb}$ : fiber laser at $1030 \mathrm{~nm}$ or a fiber Bragg grating filtered $\mathrm{Yb}$ : fiber laser at $1064 \mathrm{~nm}$. The ring cavity configuration is similar to [12-14] and the linear cavity is similar to $[15,16]$, except that the end mirror was replaced by a narrow band fiber Bragg grating, for setting up the wavelength at $1064.4 \mathrm{~nm}$.

Figure 6 shows both pulse spectra at mode locking in the two fiber lasers. The pulse width for the ring cavity was 110 fs after compression, and that for the linear cavity at $1064 \mathrm{~nm}$ was 9.4 ps without extracavity dechirp. Both experiments demonstrate that the SESAM can support the mode locking for the wavelength range from $1010 \mathrm{~nm}$ to $1064 \mathrm{~nm}$.

\section{Conclusion}

We designed and demonstrated a SESAM by phase shift

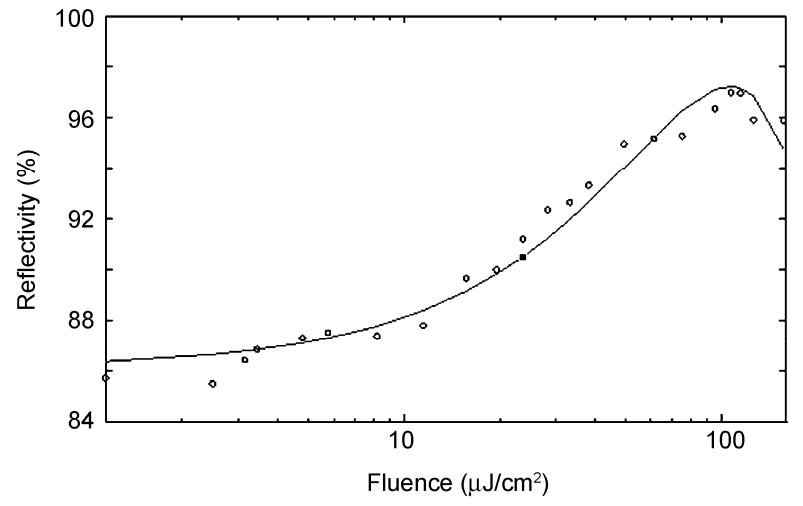

Figure 5 Measured reflectivity of the SESAM as a function of the incident laser fluence.

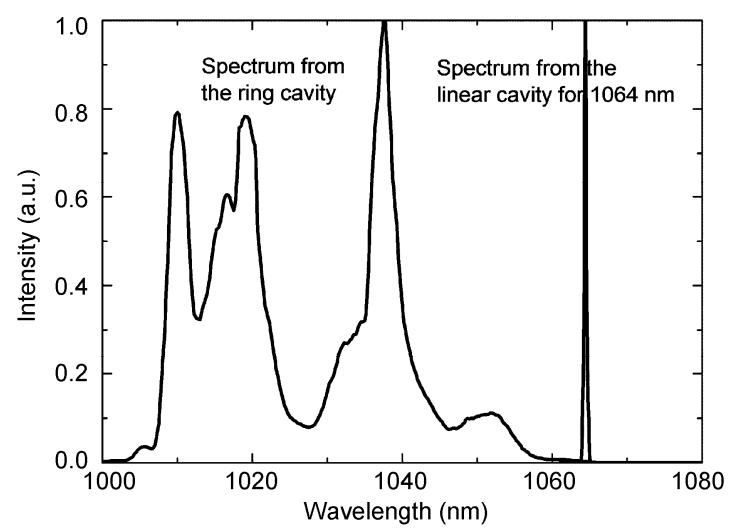

Figure 6 Pulse spectra. The broadband spectrum from a ring cavity, and the narrow sharp spectrum right at $1064.4 \mathrm{~nm}$ from the fiber Bragg grating filtered linear cavity $\mathrm{Yb}$ :fiber lasers demonstrated that the SESAM supports broadband mode locking.

compensation. The SESAM has a uniform low intensity reflectivity of $85 \%$ in the wavelength range of 1010-1080 $\mathrm{nm}$. The measurement shows that SESAM has a saturation fluence of $<30.7 \mu \mathrm{J} / \mathrm{cm}^{2}$, the modulation depth of $12 \%$, and the non-saturable loss of $3 \%$. The ring cavity and linear cavity $\mathrm{Yb}$ :fiber laser demonstrated that SESAM can support the mode locking operation in the wavelength range from 1010-1064 $\mathrm{nm}$. The design can also be extended to higher modulation depth and to other wavelength range.

This work was supported by the National Basic Research Program of China (2006CB806000), the National Science and Technology Supporting Program of China (2006BAF03A20), the National Hi-Tech Research and Development Program of China (2008AA031901), the National Natural Science Foundation of China (10974006) and Grant-in-Add from Science \& Technology Commission Haidian District, Beijing, China (K2008096).

1 Weingarten K J, Keller U, Chiu T H, et al. Passively mode-locked diode-pumped solid-state lasers that use an antiresonant Fabry-Perot saturable absorber. Opt Lett, 1993, 18: 640-642

2 Brovelli L R, Keller U, Chiu T H. Design and operation of antiresonant Fabry-Perot saturable semiconductor absorbers for mode-locked solid-state lasers. J Opt Soc Am B, 1995, 12: 311-322

3 Sun H B, Hu L Z, An H Y, et al. Generation of ultrashort optical pulse by colliding-pulse mode locking in semiconductor laser diodes. Chinese Sci Bull, 1996, 41: 1755-1755

4 http://www.batop.com/products/saturable-absorber/saturable-absorbe r-mirror/data-sheet/saturable-absorber-mirror-1040nm/saturable-abso rber-mirror-SAM-1040-10-500fs.pdf

5 Okhotnikov G, Jouhti $\mathrm{T}$, Konttinen $\mathrm{J}$, et al. 1.5- $\mu \mathrm{m}$ monolithic GaInNAs semiconductor saturable-absorber mode locking of an erbium fiber laser. Opt Lett, 2003, 28: 364-366

6 Grange R, Haim M, Paschotta R, et al. New regime of inverse saturable absorption for self-stabilizing passively mode-locked lasers. Appl Phys B, 2005, 80: 15-158

7 Spühler G J, Weingarten K J, Grange R, et al. Semiconductor saturable absorber mirror structure with low saturation fluence. Appl Phys B, 2005, 81: 27-32

8 Li J, Liu P, Lu S H, et al. Design and fabrication of erbium doped photosensitive fibers. Sci China Ser E-Tech Sci, 2009, 52: 1234-1241

9 Sun J Q, Qiu J L, Huang D X. Multiwavelength erbium-doped fiber 
laser exploiting intracavity polarization inhomogeneity. Sci China Ser E-Tech Sci, 2000, 43: 194-198

10 Palik E D. Handbook of Optical Constants of Solids. New York: Elsevier, 1998

11 Maas D J H, Rudin B, Bellancourt A R, et al. High precision optical characterization of semiconductor saturable absorber mirrors. Opt Express, 2008, 16: 7571-7579

12 Zhou C, Yang W, Zhang G, et al. Novel ring-cavity for ytterbium-doped mode-locked fiber laser incorporated with both SESAM and grating pair. IEEE Photon Tech Lett, 2009, 21: 3-5

13 Fang X H, Hu M L, Li Y F, et al. Numerical analysis of mode lock- ing in multi-core photonic crystal fiber. Chinese Sci Bull, 2010, 55: 1864-1869

14 Zhao L M, Tang D Y, Cheng T H, et al. Passive harmonic mode locking of gain-guided solitons in erbium-doped fiber lasers. Chinese Sci Bull, 2008, 53: 676-680

15 Zhang M, Chen L, Zhou C, et al. Mode-locked ytterbium-doped linear-cavity fiber laser operated at low repetition rate. Laser Phys Lett, 2009, 6: 657-660

16 Li J, Dai Y H, Zhang Z G. High repetition rate passively modelocked erbium-doped fiber laser. Chinese Sci Bull, 2008, 53: 706708

Open Access This article is distributed under the terms of the Creative Commons Attribution License which permits any use, distribution, and reproduction in any medium, provided the original author(s) and source are credited. 